\title{
A tabletop adaptive optics demonstrator for STEM outreach activities
}

Brennan Taylor, Evan Threlkeld, Tyler Brewer, J. D. Vera Cruz, Matthew Cooper

Brennan Taylor, Evan Threlkeld, Tyler Brewer, J. D. Vera Cruz, Matthew Cooper, "A tabletop adaptive optics demonstrator for STEM outreach activities," Proc. SPIE 11143, Fifteenth Conference on Education and Training in Optics and Photonics: ETOP 2019, 111431F (2 July 2019); doi: 10.1117/12.2521816 Photonics: ETOP 2019, 2019, Quebec City, Quebec, Canada 


\title{
A Tabletop Adaptive Optics Demonstrator for STEM Outreach Activities
}

\author{
Brennan Taylor ${ }^{\mathrm{a}}$, Evan Threlkeld ${ }^{\mathrm{a}}$, Tyler Brewer ${ }^{\mathrm{a}}$, JD Vera Cruz ${ }^{\mathrm{a}}$, and Matthew Cooper ${ }^{\mathrm{a}}$
}

${ }^{a}$ Air Force Research Laboratory, 3550 Aberdeen Ave SE, Kirtland AFB, NM 87112, USA

\begin{abstract}
The Air Force Research Laboratory's Laser Division faces the unique challenge of explaining the intricate technical problems in its research portfolio to a varied audience that includes upper military leadership who control program funding all the way to students of all levels as part of STEM outreach activities. An innovative solution to this challenge was developed by a team of junior scientists and engineers in the form of a series of table top demos used to visually illustrate some of the key technical challenges and fundamental science the lab is working on. One of the most challenging problems facing our team is the degrading optical effect caused by turbulence. Modern laser weapons systems utilize Adaptive Optics (AO) to correct for many of these effects and thus our team set out to showcase an AO system approachable to the public. This paper highlights how we use a commercial product as the center of our demo, lessons we have learned while showcasing this demo and a few expansion possibilities. This system boasts a unique ability to speak to all ages and thus has been used to explain key concepts to military leadership at the Pentagon as well as expose elementary students across the USA to STEM challenges. This system is one of a two-part set. The other, focusing on Line-of-Sight Stabilization, is discussed in a sister paper.
\end{abstract}

Keywords: Adaptive Optics, Laser System, Control Loop, STEM, Education, Deformable Mirror, Wavefront Sensor

\section{INTRODUCTION}

Adaptive Optics as a concept was originally invented by the astronomy community. It dates back to Cold War era research into overcoming the atmospheric disturbances seen in ground based telescope imagery of satellites. After the work was declassified in the 1990s, several other technological communities recognized key opportunities for AO systems to be integrated, including vision research, microscopy, and optical communication. The directed energy community found this technology to be extremely useful in providing optical corrections to a laser and thus has worked to shrink the associated hardware for tactical applications ${ }^{1}$. Because of the broad applications of $\mathrm{AO}$ and its unique optical components, commercially available educational kits have been created. Our demonstrator uses one such kit created by Thorlabs ${ }^{2}$.

This system utilizes a 635nm laser diode, a piezoelectric deformable mirror and an $880 \mathrm{~Hz}$ CMOS wavefront sensor to correct static disturbances and provide a visual reference sufficient to explain the fundamentals to any audience ${ }^{2}$. It is also small enough to be housed on a $2 \mathrm{ft}$ by $1 \mathrm{ft}$ optical breadboard, making it easy to transport and showcase in a variety of environments. Section 2 of this paper will explain how this demo was acquired and the story our team is trying to tell. Our team has run into several technical challenges with this system when trying to showcase some of the more advanced concepts and these efforts will be described in Section 3. Section 4 will conclude with a few expansion possibilities for consideration and how a simplified version of this lesson could be taught without an expensive demonstration kit. 


\section{Section 2. AO Demo Development and Story}

The AO demonstration was created as part of the response to a challenge presented to our original design team. We were tasked with creating an interesting, approachable tour concept for our facility. Our building is one of the most common stops for any tour group who visited the Air Force Research Laboratory at Kirtland AFB but our specific program lacked a concise way to explain our work to those interested in what we did. Our team was given one month to design and present a new tour concept that could showcase the great work we were doing to a wide variety of audiences.

From the initial stages of this project, we quickly recognized our work represents a rather technically complex and unique problem at the intersection of optics, laser physics, and aerodynamics. Very few of our potential audiences would have a scientific background in any one of these fields, much less all of them. Thus, we would need to distill the important content down to a level that, while still being technically accurate, would also be understandable by anyone regardless of their scientific background. We tossed around a few ideas for a video or storyboard but ultimately came to the conclusion that there is nothing quite like observing a scientific phenomenon occur right before your eyes. Thus, the demo tables were born. To narrow our focus for what we wanted to present, we focused on two of the most fundamental scientific challenges we face: jitter and aero-optical disturbances. This paper focuses on the second of those challenges.

Aero-optical disturbances occur when the atmosphere causes on optical distortion on light traveling through it. To an imaging sensor, such as a camera or eye, this visually appears as though the object being viewed is distorted or "twinkling" in the case of stars. This effect can be caused by broad scale atmospheric turbulence or by the turbulent structures formed around the body of a flying aircraft. Since laser weapons work by depositing energy into a small point on a target and thus causing a physical effect, maintaining the focus of the beam onto to target is paramount. If the beam passes through aero-optical disturbances without some type of correction, it becomes defocused and the power density is unable to cause the desired effect ${ }^{1}$.

This is quite a complex problem to communicate to an uneducated audience. Thus often start by reminding our audience of where they have seen this phenomenon: mirages. By starting with this common example, we can ground our discussion and walk them through how air can affect light. From there it is a simple extrapolation to connect the dots to a laser beam operating in a dynamic atmosphere. To aid in our demos, we try to keep the beam "uncorrected" as the default static setting. That way when we transition from talking about a mirage to lasers, they immediately observe a distorted beam. This distortion is often created by a simple misalignment of one of the lenses in the system, creating a defocus on the target. As the observer watches, we close the control loop between our Shack-Hartman wavefront sensor (WFS) and deformable mirror (DM) and the beam spot suddenly collapses into a bright, focused spot.

Now that we have shown the audience the effect, we transition to explaining the hardware used. We highlight this is all being done by one sensor and one correction device. Often, we first point out the WFS, describing it as a special type of camera that can sense how the light is being affected by the atmosphere. This tells us how the beam is being distorted. The software calculates a correction and sends that signal to the deformable mirror. The DM operates exactly as its name would suggest, deforming through the push and pull of tiny actuators on the backside of its face, thus applying an optical correction to the beam. As a final "wow factor" for some audience, we like to show off the calibration cycle of the DM. This steps through the various minimums and maximums of the DM, showcasing the various Zernike polynomials used to correct aberrations. For most audiences, they are impressed most by how one optic can create so many different "shapes". 


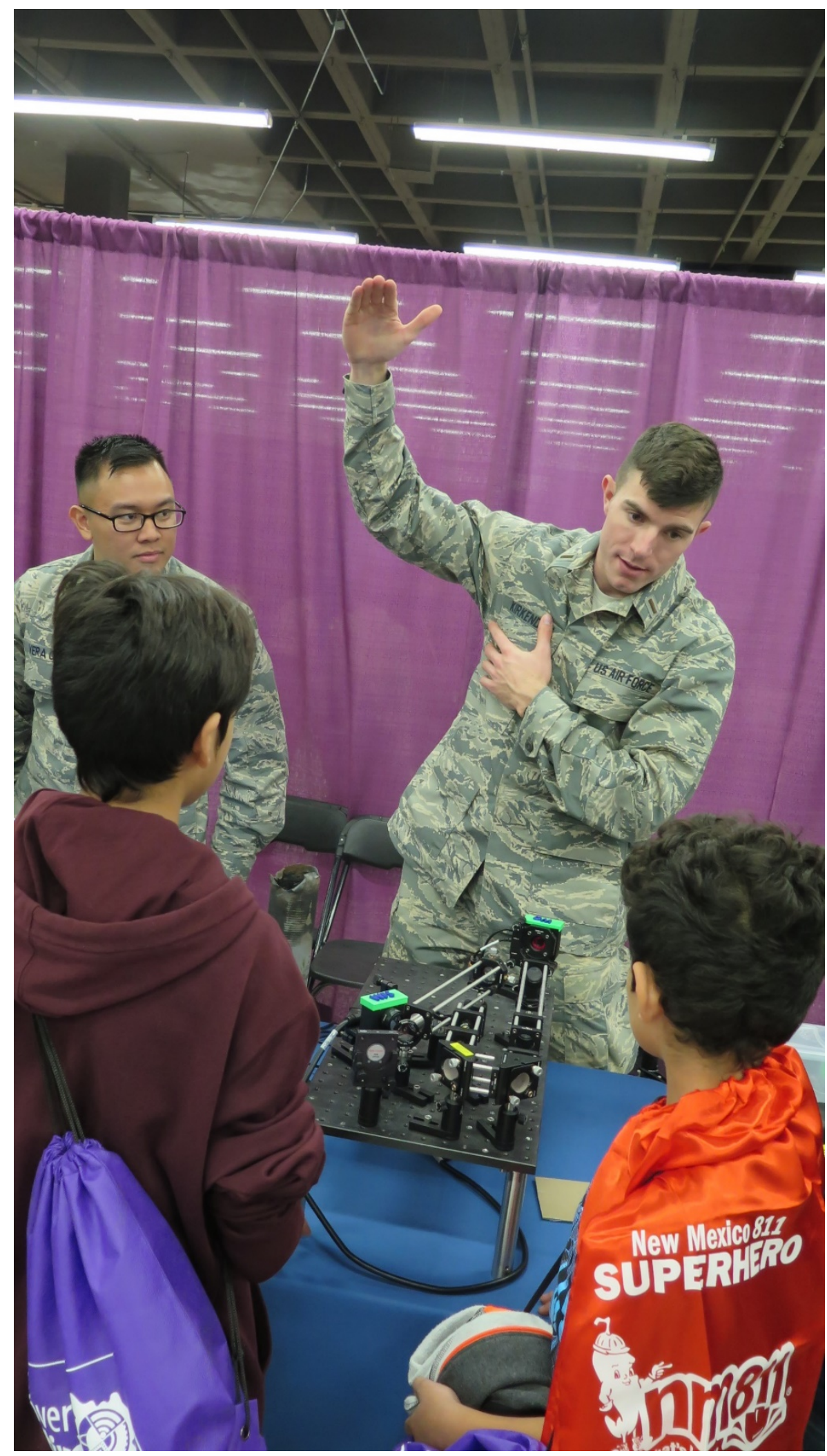

Figure 1: Lieutenants Kirkendall and Vera Cruz showcase the demo at an Albuquerque Big Brothers Big Sisters Event 


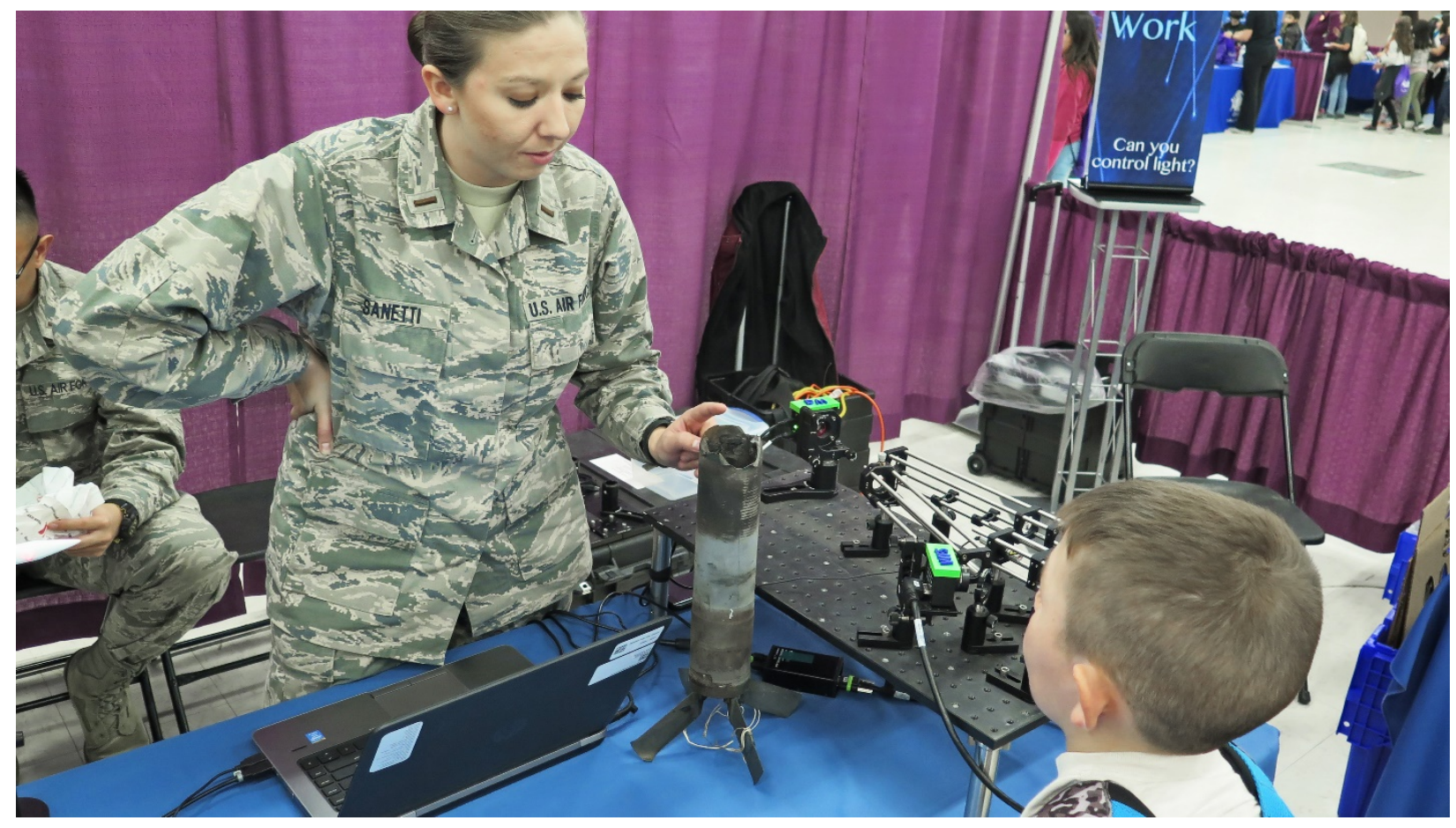

Figure 2: Lieutenant Sanetti shows students how an AO system can enable laser weapons systems

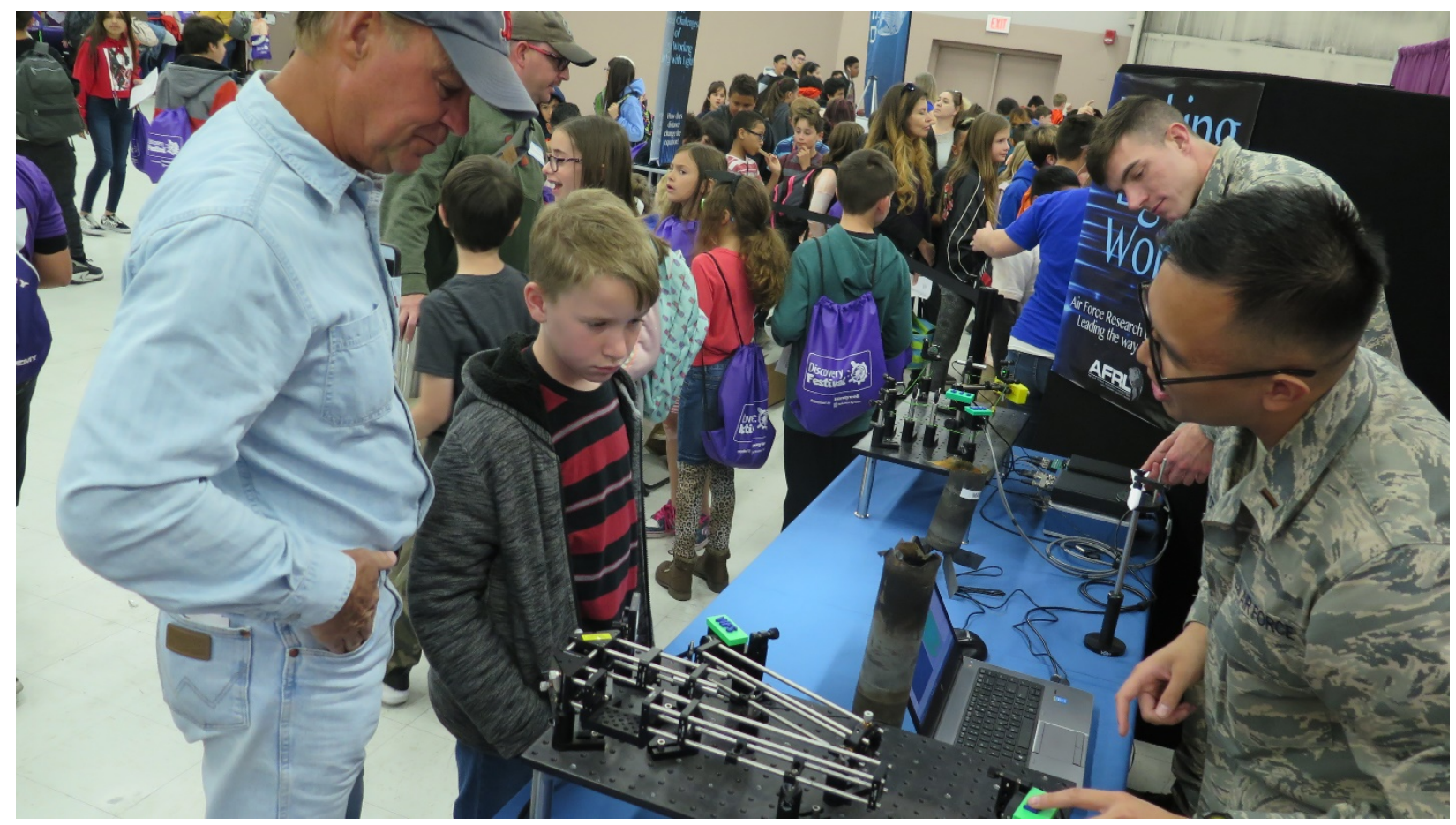

Figure 3: Lieutenant Vera Cruz shows a father and son how a deformable mirror operates 


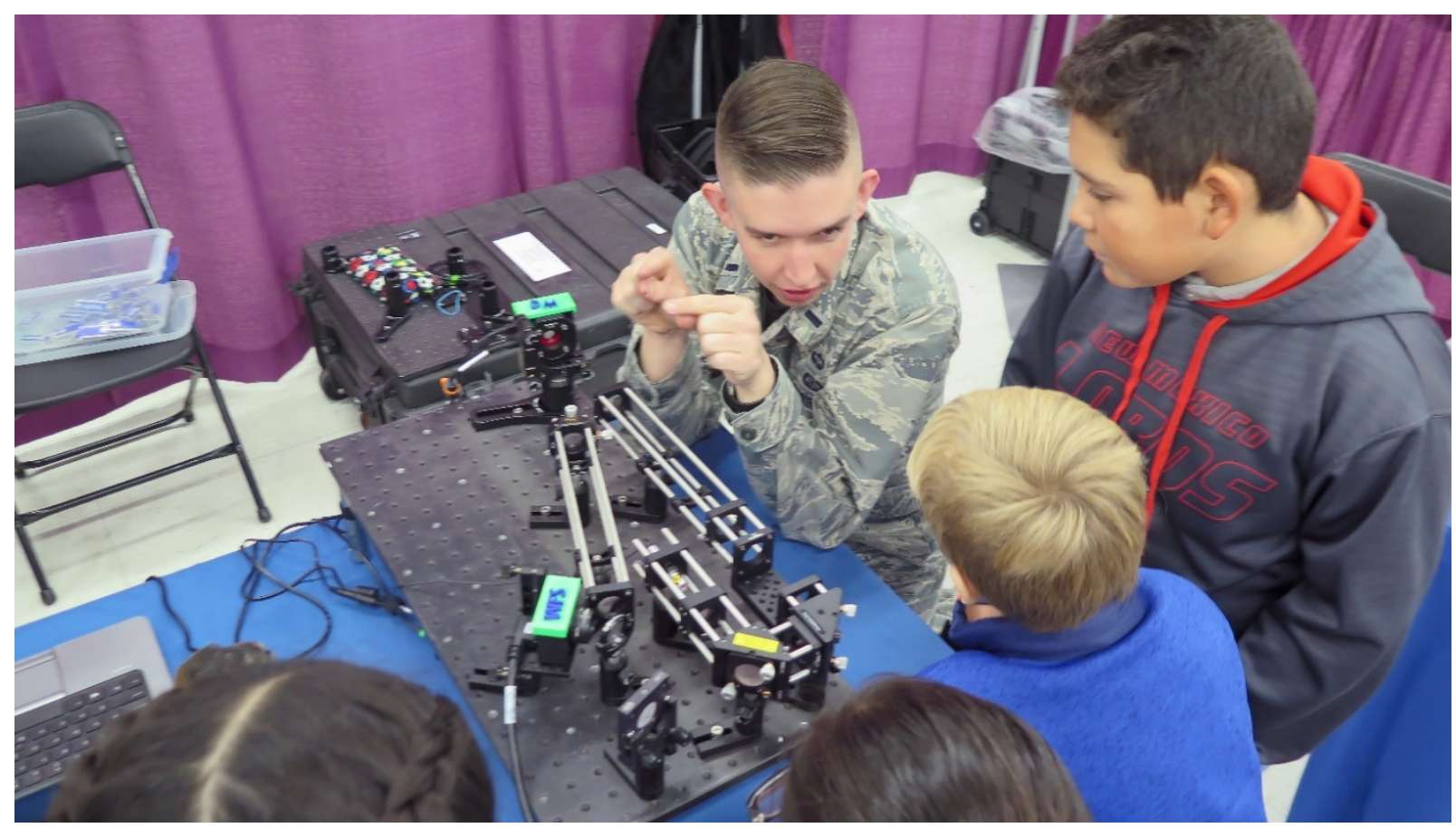

Figure 4: Lt Taylor explains the AO demo with several students at an Albuquerque event



Figure 5: Lt Brewer highlights the AO demo as part of a Making Light Work display 


\section{Section 3. System Design and Lessons Learned}

As this system is a commercial product that may be purchased by any interested user, there is little need to spell out all of its technical details in this medium. Our team did encounter a few technical challenges when building and first working with this demo that are worth explaining.

Immediately upon setting up this system and first confirming the control loop would close, our team was quite enthusiastic about throwing all sorts of aero-optical disturbances in front of the system. A series of phase screens were our first test and they all created a barely visible disturbance that was also correctable via the DM. However, as previously mentioned, our goal for this effort was large scale visibility for a wide audience range. Thus, these small phase aberrations were not going to be enough to satisfy. In our excitement, we seized upon another piece of laboratory hardware that could generate large scale atmospheric density gradients: a heat gun. By directing the output stream of our heat gun along the beam path we were able to see significant disturbances in the beam with the unaided eye. However, we quickly realized the frequency content of those disturbances was too fast for our control loop to be able to correct. A quick look back into the supporting documentation confirmed a maximum closed loop operating frequency of up $190 \mathrm{~Hz}$. Thus we had bounded our problem: the disturbance of a static phase screen was practically invisible to the eye, while the disturbance created by a heat gun, while clearly visible, was uncorrectable. Our first, and admittedly inelegant, solution was to simply misalign one of the optics. This intentional shift in part of a beam expander created a defocus on the beam and thus was clearly visible and easily correctable. While this worked for our first few demo events, it created a much larger need for "hand waving" over some of the more technically accurate questions and pulled our demo further away from scientific accuracy than we were comfortable with. What followed was a six month process during which we destroyed countless plastic CD containers attempting to manufacture our own custom "phase screens" with an appropriately medium level of optical disturbance - enough to see unaided but also not so much as to break our control loop. In the end, we realized that while we would be given high marks for creativity and effort, optical manufacturing was not our skill set. The solution presented itself in the form of a discussion with a few Thorlabs employees at the Photonics West trade show: eyeglasses. They had run into the same challenge of easily displaying a static disturbance and had eventually settled on using lenses from prescription eyeglasses. After a few calls to a local optometrist, we were able to secure a few old and unwanted lenses of varying prescriptions that accomplished our goal: a visible disturbance, fully correctable by the AO system!

\section{Section 4: System Expansion and Modifications}

As our demonstration is nearly entirely an off the shelf demonstration product, simple easy modifications are not as readily apparent. We have previously discussed a small addition of various disturbance generation sources. The one other addition we have made to the system is entirely aesthetic but has made an absolutely massive difference! We bought a smoke machine. By adding a small smoke or fog machine underneath the breadboard and allowing the haze to drift up through the holes in the board, the beams of the laser become visible to the unaided eye. This allows the audience to observe how the beam expands, focuses, and reflects through the system, creating an unbelievably eye-catching display! This simple addition has wowed audiences from senior executives to school children alike and is highly recommended by our team. We should note that with the addition of our smoke machine, we also added a clear window in front of the DM and WFS to attempt to keep residue from collecting on those sensitive electronics. So far we have found that even after a multi-hour event, only a minimal amount of residue is found on our optics and we typically clean the system after every 2-3 events.

For the highly experienced user, with plenty of funding for expanding capabilities, our team was told Thorlabs is working on a new version of this system that will come with two deformable mirrors to work in a "woofer-tweeter" design to compensate for a broader spectrum of disturbances. This system would even allow for the operator to intentionally deform the beam in recognizable ways, such as making it a "smiley face", to further show the capabilities and possible extensions of the system. 
Finally, we wanted to address opportunities to simplify this demo and present the same lesson without a \$10k demo. There is a bit of a challenge here. The fundamental principle of air being able to distort light is simplicity itself to showcase. However, it's the correction and sensing capability that presents quite a technical challenge. For advanced students with a geometry background, the ideas behind a lenslet array and wavefront reconstruction could offer an interesting practical application problem. Similarly, the fundamentals of a DM could be accessible to an advanced physics class. However, without a dramatic simplification of some kind, most students below secondary level will have trouble grasping these ideas without a visual aid. This is an area where our team has spent a fair amount of time and energy without much success. We have settled on the idea of trying to develop a "DIY DM". That is a large scale, userhand-manipulated, deformable mirror surface which would allow audience members to see "behind the curtain" of how a DM operates. Our idea was born from playing with a 2D basic ray-optics demo. This simplest version would be to simply have a segmented mirror that the user can physically steer and observe how the various rays reflect. However, our team desires to come up with a design that will showcase not only the function of the deforming mirror surface, but also highlight the mechanism that creates the deformation in a simple observable way. That is the engineering challenge we are stuck on as of the writing of this paper. This might be a worthwhile challenge for interested students as an enrichment activity after a ray optics and light lesson.

\section{REFERENCES}

[1] Duffner, W. and Fugate, Q., [The Adaptive Optics Revolution: A History], University of New Mexico Press, Albuquerque, (2009).

[2] Thorlabs, Inc., “Adaptive Optics Kits”, Thorlabs Inc., 2019,

<https://www.thorlabs.com/newgrouppage9.cfm?objectgroup_id=3208> (3 May 2019). 